\title{
PUBLICAÇÃO ACADÊMICA INTERNACIONAL COMO ESTRATÉGIA DE INTERNACIONALIZAÇÃO DAS INSTITUIÇÕES DE ENSINO SUPERIOR
}

\author{
LUISA BIAVA \\ Universidade Federal de Santa Catarina \\ luisa.biava@ufsc.br
}

\begin{abstract}
RESUMO
Este artigo tem como objetivo apresentar uma análise das publicações acadêmicas internacionais dos pesquisadores da Universidade Federal de Santa Catarina (UFSC). Em um primeiro momento, é identificada a publicação internacional como fator de avaliação da internacionalização das universidades, com base nas literaturas internacional e nacional. Após esse diagnóstico, buscou-se analisar a publicação científica dos pesquisadores da Universidade Federal de Santa Catarina, no ano de 2015, em periódicos internacionais. Para tanto, foi utilizado um sistema extrator do Currículo Lattes (base de dados do Conselho Nacional de Desenvolvimento Científico e Tecnológico - CNPq). A pesquisa foi bibliográfica, documental e descritiva, e a análise foi realizada com base em um estudo quantitativo. Como resultado, buscou-se a divulgação e o desenvolvimento da produção científica internacional por meio da exposição de indicadores das características das publicações internacionais da UFSC em 2015. Foi possível concluir que os pesquisadores publicaram um número expressivo de artigos em periódicos internacionais, estando os Estados Unidos como o país com o maior número dessas publicações, e a maior parte dos pesquisadores com um artigo publicado internacionalmente.
\end{abstract}

PALAVRAS-CHAVE: publicação científica; internacionalização; Instituições de Ensino Superior. 


\section{INTRODUÇÃO}

A globalização traz um novo paradigma para as universidades em todo o planeta. Assim como outras organizações, as Instituições de Ensino Superior (IES) estão sendo influenciadas pela globalização e passam a adotar medidas para se internacionalizarem. A inserção da universidade no cenário global é uma necessidade, mas também uma consequência do ambiente dinâmico em que ela se encontra.

Não só o intercâmbio acadêmico, presente na universidade desde a sua criação, mas também as ações de mobilidade estudantil, de cooperação internacional, de equipes multilíngues, de pesquisas interinstitucionais estão movendo as universidades para um caminho definitivo - a internacionalização.

Nesse contexto, a universidade, tendo como marca a produção do conhecimento, sempre buscou a internacionalização da função pesquisa, mesmo que apenas por iniciativa do próprio pesquisador. A internacionalização configura-se como uma marca das relações entre as universidades (MOROSINI, 2006).

Ribeiro (2000) defende que a globalização desperta nas Instituições de Ensino Superior uma adaptação organizacional estratégica frente a esse fenômeno de transformações contínuas. Essas estratégias envolvem maiores vínculos de pesquisa transnacionais e redes, associações e outros acordos entre instituições, entre professores, entre pesquisadores e/ou estudantes, com ajuda das tecnologias de informação e comunicação.

Paralelamente a isso, a publicação científica apresenta-se como uma das formas principais de divulgação do conhecimento produzido nas universidades, além de ser considerada fundamental para a progressão da carreira dos professores de Ensino Superior, servindo como fator de avaliação dos docentes. Nesse sentido, a publicação é um instrumento necessário para a atividade científica, para a disseminação do conhecimento, tanto para a comunidade científica quanto para a sociedade em geral, que muitas vezes é beneficiada pelas pesquisas realizadas nas universidades.

A publicação de artigos em periódicos e revistas internacionais é cada vez mais valorizada nas universidades, pois além de divulgar a pesquisa realizada, também projeta a instituição no âmbito internacional. Diante disso, este artigo tem como objetivo discorrer sobre a análise da publicação científica realizada pelos pesquisadores da Universidade Federal de Santa Catarina em periódicos internacionais no ano de 2015.

\section{INTERNACIONALIZAÇÃO DA EDUCAÇÃO SUPERIOR}

Assim como outros tipos de organizações, as Instituições de Ensino Superior vêm sentindo os efeitos da globalização, que desencadeia diferentes mudanças. Para Ribeiro (2000), mesmo sendo baseadas em princípios tradicionais, as universidades foram criadas para a continuidade e não estão imunes às transformações do ambiente globalizado. É necessário que essas instituições adaptem-se estrategicamente a esse desenvolvimento, a fim de tornarem-se menos resistentes a mudanças e mais capazes de adaptarem-se aos novos paradigmas ou, mais ainda, de transformarem-se para melhor.

O caráter universal da educação superior e a busca por avanços baseiam-se na livre circulação de ideias por meio dos campos científicos e das disciplinas acadêmicas. No Brasil não é diferente, a universidade precisa compreender que está envolvida em um projeto global. O conhecimento que circula dentro da universidade já não cabe mais nas fronteiras da instituição, nem do país, e, por isso, a universidade brasileira precisa fazer parte do saber internacional (UNESCO, 2003). 
A educação superior, com seu caráter de geração e divulgação de conhecimento, proporciona uma oportunidade para os países em desenvolvimento de superarem as diferenças que os separam dos países desenvolvidos. Para a UNESCO (2003, p. 102), “esses países precisam fortalecer seu treinamento de alto nível e a capacidade de pesquisa, de modo maciço e com toda urgência, se querem evitar a marginalização e exclusão".

Corroborando com isso, Gacel e Ávila (2009) defendem que os países da América Latina precisam urgentemente elevar os níveis educacionais, seguindo as tendências globais. Frente a esse objetivo, as estratégias de internacionalização mostram-se fundamentais, uma vez que, mediante a cooperação internacional, é possível diminuir o atraso e a lacuna que distancia esses países das nações desenvolvidas.

Nesse sentido, diante do processo de globalização e da visão dessa necessidade de transformação, a internacionalização foi inserida gradativamente na educação superior a partir da década de 1990. De acordo com Ribeiro (2000), esse processo é consequência do caráter mundial do ensino e da pesquisa, o qual se fortalece com a integração de instituições e países, consequência também da natureza mundial das comunicações nos dias de hoje.

Morosini (2006) identifica dois momentos distintos na internacionalização da educação superior: primeiro, a criação do conceito de internacionalização e sua complexidade; segundo, a procura por estratégias de implantação e melhoria da internacionalização - que, para a autora, estão ligadas a um processo estratégico de globalização e de regionalização das sociedades e o consequente impacto desses processos na educação superior.

Tratando-se de um conceito complexo, não há uma definição exata para a internacionalização, a qual é uma característica intrínseca da ciência e da tecnologia, que resulta da dimensão internacional dos múltiplos atores, processos, resultados e impactos da pesquisa científica e do desenvolvimento tecnológico. Parece, de acordo com a literatura, que a internacionalização da ciência e da tecnologia acontece de forma espontânea na formação e na organização das comunidades científicas, na produção e difusão de conhecimento (MANUAL DE SANTIAGO, 2007).

A internacionalização, de acordo com Knight (2012), precisa ser integrada de maneira sustentável às principais funções da educação superior - o ensino, a pesquisa e a produção do conhecimento - para melhor servir a sociedade. A autora acredita que esse é um processo adaptável que tem como finalidade a conexão entre a dimensão internacional, intercultural e global e os objetivos, funções e atividades do Ensino Superior.

Nesse sentido, as universidades têm estabelecido estratégias para a gestão da internacionalização, as quais impulsionam tanto a participação de estudantes, docentes e investigadores em redes internacionais, quanto a sua associação a organizações que promovem a cooperação internacional universitária (GACEL; AVILA, 2009).

Seguindo essa perspectiva, Stallivieri (2004) afirma que as universidades exercem um papel fundamental na cooperação internacional, a qual passa a fazer parte da vida acadêmica, na medida em que possibilita a troca constante de informações entre grandes centros, originando redes de integração, diminuindo barreiras e aprimorando o desenvolvimento científico, tecnológico, social e cultural.

Essa integração, de acordo com Stallivieri (2004), incentiva a mobilidade acadêmica, tanto de alunos, quanto de professores, pesquisadores e gestores das universidades, permitindo a criação de projetos de pesquisa, a troca de informações e a viabilidade de publicações científicas, as quais, além de projetarem a instituição em nível mundial, tornam possível a transmissão do conhecimento para além das fronteiras nacionais. 


\subsection{A Publicação Científica Internacional}

Diante desse panorama de internacionalização da educação superior, a publicação científica apresenta-se como uma forma de divulgação do conhecimento adquirido e da própria instituição. Stallivieri $(2009$, p. 35) defende que as universidades devem "produzir e publicar cientificamente os resultados das investigações em revistas e periódicos científicos de renome mundial".

A publicação é vista, no cenário global, como uma forma rápida de transferência de conhecimentos e tecnologias. Silva (2007) afirma que o fluxo de conhecimento derivado de pesquisas é intenso e possibilita que novas ligações e acordos de cooperação sejam realizados. Não só os países em desenvolvimento tem interesse em participar de projetos de pesquisa com países desenvolvidos, mas o oposto também acontece quando há cooperação internacional.

Sebastián (2009) argumenta que as universidades latino-americanas são cobradas em avaliações e medições de produtividade, de publicações e citações, o que tem sido forte em alguns conselhos nacionais de universidades ou de pesquisa. $\mathrm{O}$ caráter individual da produção científica, hoje passa a ser coletivo, realizado principalmente por grupos de pesquisa e de colaboração de diferentes instituições e países. Esses grupos podem formar redes de pesquisa nacionais e internacionais, o que tem se mostrado uma tendência crescente nas ciências sociais, no desenvolvimento tecnológico e na inovação.

Sebastián (2003) acredita que um dos motivos para a cooperação entre pesquisadores de diferentes países é a necessidade de complementação das capacidades, que advém da especialização dos grupos de pesquisa, do caráter multidisciplinar das abordagens, da fusão de campos científicos em novas tecnologias e da disparidade dos processos de inovação.

Além desses motivos, Sebastián (2003) aponta outras questões relacionadas à abertura política, social e cultural e ao acesso cada vez maior aos meios de comunicação, principalmente a internet. Nas universidades latino-americanas, a internacionalização da pesquisa aparece por meio das relações dos professores e pesquisadores com instituições estrangeiras em que realizam a sua formação, da mobilidade estudantil, da realização de projetos em parceria e da participação em redes de investigação.

Para López (2015), um dos fatores que contribuem para o avanço das atividades científicas no cenário internacional é o desenvolvimento das disciplinas, as quais atravessam as tendências do aumento da especialização disciplinar e do aumento de campos interdisciplinares. Essas tendências incentivam o contato e a cooperação entre diferentes países, os quais geram os avanços científicos mais significativos. A autora destaca as políticas de financiamento que estimulam a formação de grupos de trabalho e fomentam a colaboração internacional e a globalização da ciência e tecnologia.

Nesse contexto, López (2015) defende que a cooperação científica está conectada à geração de conhecimento relevante, dando utilidade social e visibilidade internacional às investigações na América Latina. $O$ fenômeno da internacionalização da ciência relaciona-se aos sistemas de avaliação e às políticas científicas que impulsionam direta ou indiretamente as dimensões internacionais da pesquisa. Para Sebastián (2003), o alvo principal das publicações é a própria comunidade científica, mas também pode envolver as comunidades locais, usuários e beneficiários dos avanços da ciência e da tecnologia, tanto em níveis nacionais como globais.

$\mathrm{Na}$ esfera internacional, a metodologia adotada para a avaliação da qualidade da educação superior é baseada em rankings acadêmicos. Vieira e Lima (2015) apontam que as avaliações são baseadas em citações científicas, prêmios, publicações em periódicos renomados, entre outros aspectos analisados. As universidades americanas e britânicas lideram os rankings internacionais como o Times Higher Education (THE) e o QS World 
University, principalmente pela representatividade nos prêmios Nobel (VIEIRA; LIMA, 2015).

Os rankings internacionais tornaram-se ferramentas de avaliação da educação superior e de sua internacionalização e, como consequência, um incentivo para a produção de conhecimento, para a atração de estudantes e de professores internacionais e para o financiamento de pesquisa (DELGADO-MÁRQUEZ; HURTADO-TORRES; BONDAR, 2011).

Apesar de amplamente considerados, os rankings internacionais são criticados por não ponderarem os diferentes contextos em que as universidades estão inseridas. Seja em países desenvolvidos ou em desenvolvimento, todos são avaliados pelos mesmos critérios. A internacionalização é considerada nos rankings universitários principalmente pela quantidade de publicações com coautoria internacional e pelo número de mobilidade estudantil de cada universidade.

O desenvolvimento científico e tecnológico sempre teve uma dimensão internacional, associada à própria dinâmica da formação dos pesquisadores, à natureza dos processos de investigação e à difusão do conhecimento científico e à geração e transferência de tecnologia. A internacionalização favorece a formação e a especialização de professores e pesquisadores, já que amplia o âmbito de atuação e a qualidade dos processos, gerando fluxos de colaboração que permanecem ao longo do tempo, mesmo após o término da formação no exterior (MANUAL DE SANTIAGO, 2007).

Hoje, a internacionalização envolve também processos sociais e econômicos relacionados à utilização do conhecimento científico. Os cenários da economia e do mercado, a busca por processos sociais e ambientais sustentáveis conduzem a uma revalorização da cooperação e da internacionalização da ciência e da tecnologia. Diante disso, a produção científica tem como objetivo principal o aumento do conhecimento e a contribuição conceitual e metodológica para o desenvolvimento de novas pesquisas, que podem colaborar para a solução e o alívio de problemas sociais e para o desenvolvimento econômico.

\subsubsection{A publicação científica internacional no Brasil}

Para Bozu e Herrera (2009), ser um pesquisador é uma das competências profissionais do professor de Ensino Superior, por meio da qual possibilita a produção e a difusão de conhecimento, além de melhorar a qualidade da docência. Nesse sentido, sendo um dos requisitos da evolução da carreira docente a publicação dos resultados de suas pesquisas e a procura por periódicos vem ultrapassando as fronteiras do país. Serra, Fiates e Ferreira (2008) defendem que publicar no Brasil já é difícil, tornando a publicação em periódicos internacionais ainda mais elaborada.

Por meio da publicação científica, os pesquisadores de uma universidade podem expor o resultado final de suas pesquisas, a fim de que seja não só socializado, mas também avaliado por outros pesquisadores e pela comunidade científica (VARGAS; FÉLIX; MATOS, 2004). Para Serra, Fiates e Ferreira (2008), “os artigos publicados em revistas científicas são por excelência os meios pelos quais a comunidade científica divulga e agrega conhecimento a uma determinada área”. Por esses e outros motivos, a publicação científica é um requisito importante na evolução da carreira de professores e pesquisadores.

Soares e Casa Nova (2015) apontam que, no Brasil, a Coordenação de Aperfeiçoamento de Pessoal de Nível Superior (Capes), fundação do Ministério da Educação (MEC), avalia os programas de pós-graduação e tem, entre os principais critérios de avaliação, a quantidade e a qualidade das publicações científicas dos pesquisadores. Uma das missões da Capes é a promoção da cooperação científica internacional, que envolve, entre outros aspectos, a publicação de pesquisas brasileiras em periódicos internacionais. 
A avaliação da Capes utiliza critérios de classificação para os periódicos brasileiros e internacionais, o sistema Qualis-Periódicos, utilizado também para avaliar a produção científica dos programas de pós-graduação (CAPES, 2016). A Capes também avalia a internacionalização dos programas mediante a análise da participação dos docentes em publicações, em associações internacionais, na organização de eventos internacionais e da mobilidade estudantil (VARGAS, FÉLIX, MATOS, 2004).

Em seu Plano Nacional de Pós-Graduação (PNPG) para os anos de 2011 a 2020, a Capes faz um exame da produção científica como medida da presença da ciência brasileira no âmbito internacional, além de comentar as ações e mecanismos de cooperação internacional e a sua relação com as publicações (PNPG, 2010). De acordo com o PNPG, o número de artigos científicos publicados em revistas indexadas tem crescido consideravelmente nos últimos anos, estando muito acima da média mundial. Em 2009, o Brasil ocupava a $13^{\mathrm{a}}$ posição em número de artigos publicados em bases mundiais (PNPG, 2010).

O PNPG analisa, quanti e qualitativamente, o número e o impacto dos artigos publicados pelos pesquisadores brasileiros entre 2004 e 2008. Algumas áreas são destaques, como engenharia, matemática e física, estando próximas das médias mundiais. Além disso, o documento traz uma avaliação da atuação de organizações brasileiras no cenário mundial, identificando um grande número de envolvimento em eventos e organismos internacionais.

Como recomendações, a Capes considera fundamental a busca pela geração de novos conhecimentos como base para a expansão dos programas de pós-graduação das universidades. Esse objetivo pode ser alcançado com uma maior integração entre instituições brasileiras e internacionais, que promovam o crescimento da ciência e o protagonismo do Brasil no cenário mundial. As sugestões destacadas no PNPG (2010) mostram-se ligadas principalmente ao envio de estudantes a outros países, à captação de alunos e pesquisadores visitantes estrangeiros e ao aumento de publicações com organizações internacionais.

Outro órgão de fomento à pesquisa científica e tecnológica é o Conselho Nacional de Desenvolvimento Científico e Tecnológico ( $\mathrm{CNPq}$ ), agência do Ministério da Ciência, Tecnologia e Inovação (MCTI). Uma das competências da Instituição é "promover a realização de acordos, protocolos, convênios, programas e projetos de intercâmbio e transferência de tecnologia entre entidades públicas e privadas, nacionais e internacionais" (CNPQ, 2016).

A Coordenação-Geral de Cooperação Internacional (CGCIN) do CNPq tem como missão "fortalecer e aperfeiçoar a colaboração internacional em Ciência, Tecnologia e Inovação, mobilizando competências no Brasil e no exterior, contribuindo para a qualificação de pessoas e promovendo pesquisa, desenvolvimento e inovação". A CGCIN, entre suas ações, apoia a mobilidade de pesquisadores para o desenvolvimento de pesquisas, a formação e a capacitação de brasileiros em outros países e de estrangeiros no Brasil, além de financiar pesquisas e visitas científicas, e participar de projetos e programas de apoio ao desenvolvimento científico e tecnológico com outras agências de cooperação internacional (CNPQ, 2016).

No que tange à cooperação internacional, a Agência Brasileira de Cooperação $(A B C)$, do Ministério das Relações Exteriores (MRE), tem como atribuição "negociar, coordenar, implementar e acompanhar os programas e projetos brasileiros de cooperação técnica, executados com base nos acordos firmados pelo Brasil com outros países e organismos internacionais". A ABC representa o governo brasileiro e coordena negociações de diferentes programas e projetos com parceiros bilaterais, regionais e multilaterais de interesse público, tornando possível o acesso a tecnologias, conhecimentos, informações e capacitações de maneira mais ágil (ABC, 2016). 


\subsubsection{A publicação científica e a internacionalização na UFSC}

Dentro das universidades, a internacionalização deve ser adotada como política e considerada em todas as áreas da instituição. Ribeiro (2000) defende que esse processo acontece como uma abertura institucional e precisa integrar o plano estratégico e as políticas gerais das instituições de Ensino Superior. Nesse sentido, faz-se necessário uma política institucional clara e definida de internacionalização.

No âmbito da Universidade Federal de Santa Catarina (UFSC), as ações de internacionalização são articuladas pela Secretaria de Relações Internacionais e envolvem o ensino de graduação, pós-graduação, a pesquisa e a extensão, a fim de elevar a qualidade acadêmica (SINTER, 2016). A UFSC tem como política de internacionalização promover a excelência científica e tecnológica do país e proporcionar a solidariedade entre os povos.

Documento em que são definidas as politicas, diretrizes e estratégias da instituição, o Plano de Desenvolvimento Institucional (PDI) da UFSC menciona a internacionalização não só em seus objetivos, mas também na missão e nos valores da Universidade. A missão da UFSC, de acordo com o PDI 2015-2019 (2015, p.22), é:

\footnotetext{
produzir, sistematizar e socializar o saber filosófico, científico, artístico e tecnológico, ampliando e aprofundando a formação do ser humano para o exercício profissional, a reflexão crítica, a solidariedade nacional e internacional, na perspectiva da construção de uma sociedade justa e democrática e na defesa da qualidade da vida.
}

Um dos valores levantados pelo PDI 2015-2019 (2015, p.23) é a internacionalização. A UFSC busca ser "capaz de intensificar parcerias e convênios com instituições internacionais, contribuindo para o seu desenvolvimento, o do Brasil e o de outras nações". Pode-se constatar, portanto, que a Universidade possui uma política de internacionalização instituída nos documentos e órgãos oficiais da instituição.

O Plano de Desenvolvimento Institucional da UFSC (PDI 2015-2019, p. 43) traz como objetivo, no âmbito da pesquisa, ampliar a internacionalização das atividades da Universidade, o que envolve as seguintes metas, já estabelecidas também no PDI anterior:

a) incrementar ações e projetos de cooperação internacional;

b) fomentar a cooperação institucional, interinstitucional, nacional e internacional em redes de pesquisa, principalmente as de alta complexidade;

c) fomentar a participação de docentes, discentes e servidores técnico-administrativos em eventos científicos internacionais para apresentação de trabalhos;

d) ampliar a publicação em revistas indexadas em bases de referência internacional;

e) incentivar o intercâmbio internacional do corpo discente e programas de dupla titulação e de cotutela.

O PDI 2010-2014 (2014, p. 106) trazia grandes metas para a Secretaria de Relações Internacionais da UFSC, abordando assuntos como tornar a universidade de classe mundial, mobilidade acadêmica, aumentar a visibilidade da UFSC internacionalmente, intensificar a articulação com outras instituições e universidades internacionais, tornar efetivos os convênios internacionais, captar recursos para mobilidade estudantil, gerenciar a cooperação acadêmica e o intercâmbio.

Já, o PDI anterior da Universidade abordava outras questões de internacionalização e dados institucionais, incluindo a quantidade de publicação científica internacional dos pesquisadores da UFSC (PDI 2004-2009, 2004). Em 2003, foram 373 artigos publicados em periódicos internacionais indexados, equivalendo a $34 \%$ do total de artigos publicados. Nessa 
época, de acordo com o Relatório de Gestão (2003), a UFSC contava com o numero de 1.555 docentes de Ensino Superior.

Em outros documentos, o número de publicações em periódicos internacionais não é mencionado, principalmente nos últimos anos. Diante do exposto neste referencial teórico, essa falta de informações configura-se como prejudicial no ponto de vista da gestão da universidade, visto que os dados sobre a produção científica e, consequentemente, a respeito das publicações internacionais das universidades mostra-se como fundamental para os processos de internacionalização da educação superior.

Nesse sentido, a pesquisa que aqui se propôs buscou evidenciar os indicadores da publicação internacional da Universidade Federal de Santa Catarina, podendo servir de subsídio na tomada de decisões sobre a pesquisa e a internacionalização da instituição de educação superior estudada.

\section{PROCEDIMENTOS METODOLÓGICOS}

O presente artigo caracteriza-se como um estudo exploratório, uma vez que se buscou na bibliografia pesquisada e nos documentos selecionados, obter mais informações a respeito da internacionalização da educação superior e das publicações científicas no âmbito mundial, nacional e institucional. De acordo com Moreira e Caleffe (2008), as pesquisas exploratórias têm como objetivo aproximar o pesquisador de determinado fenômeno, constituindo o primeiro passo de um estudo, podendo se constituir de um levantamento bibliográfico.

Após a análise do referencial teórico, foi realizada uma pesquisa descritiva, a qual, segundo Santos (2004), trata-se de um levantamento das características de um fato ou processo analisado. A pesquisa descritiva baseia-se na análise e na descrição de um fenômeno (MOREIRA; CALEFFE, 2008), de modo que a aqui descrita foi realizada a partir da análise das publicações científicas dos pesquisadores da UFSC no ano de 2015.

Para Rudio (2003), a pesquisa descritiva é aquela em que o pesquisador busca conhecer e descrever uma realidade, mas não interfere nela, apenas observa, descreve e classifica o fenômeno e os processos que o compõem. Essa análise foi realizada com base em uma coleta de dados concretizada com a utilização da ferramenta IPÚ (a fonte, em tupiguarani), um sistema da Superintendência de Governança Eletrônica e Tecnologia da Informação e Comunicação da Universidade Federal de Santa Catarina.

O objetivo do sistema é extrair os currículos dos membros da UFSC da Plataforma Lattes, banco de dados do Conselho Nacional de Desenvolvimento Científico e Tecnológico (CNPq), padrão nacional de registro da produção acadêmica de estudantes e pesquisadores do Brasil. Os dados são extraídos semanalmente e são dispostos por meio de ferramentas de busca e gráficos, que ficam disponíveis à comunidade da Universidade.

Coletados os dados do sistema de informação, a análise foi feita de forma quantitativa, a que é empregada quando é possível obter os dados numéricos e mensurar com base em estatísticas (MOREIRA; CALEFFE, 2008). Foram utilizados, como base de dados, todos os registros de artigos publicados por pesquisadores da UFSC, disponibilizados na Plataforma Lattes referentes ao ano de 2015, totalizando um universo de 5.102 registros.

O estudo limitou-se a analisar as publicações internacionais do ano de 2015 , sendo que aqueles pesquisadores que não adicionaram as publicações ao seu currículo, na Plataforma Lattes, não apareceram na análise, já que esta Plataforma é uma base de dados de autopreenchimento. Além disso, não foi possível realizar uma análise detalhada da classificação e qualidade dos artigos e periódicos em que foram publicados. 


\section{A PUBLICAÇÃO INTERNACIONAL NA UFSC}

A análise das publicações em periódicos internacionais dos pesquisadores da Universidade Federal de Santa Catarina no ano de 2015 foi baseada nos registros feitos por eles na Plataforma Lattes do CNPq. O total de artigos registrados foi de 5.102, sendo que o mesmo artigo pode ter sido registrado por mais de um pesquisador, o que acontece quando a publicação parte de uma equipe.

$\mathrm{Na}$ primeira análise, é importante salientar o número de artigos e periódicos registrados pelos pesquisadores da UFSC, que retirando os artigos duplicados, ou seja, registrados em mais de um currículo, totalizam 3.996 artigos. Desses, 2.121 foram publicados em revistas e periódicos brasileiros e 1.875 em revistas e periódicos de outros países.

O número de artigos publicados no Brasil e em outros países em comparação com a quantidade de periódicos em que foram feitas essas publicações pode ser visualizado na Tabela 1. Os 2.121 artigos publicados no Brasil foram em 1.104 revistas ou periódicos brasileiros, o que equivale a $53,1 \%$ dos artigos em $48,2 \%$ dos periódicos. O número de artigos publicados em revistas internacionais em 2015 chegou a 1.875, em 1.188 diferentes revistas. Isso equivale a $51,8 \%$ do total de periódicos nacionais e internacionais envolvidos nas publicações.

Tabela 1 - Artigos publicados

\begin{tabular}{l|cc|cc}
\hline & \multicolumn{2}{|c|}{ Artigos } & \multicolumn{2}{c}{ Periódicos } \\
\hline Brasil & 2121 & $53,1 \%$ & 1104 & $48,2 \%$ \\
Outros Países & 1875 & $46,9 \%$ & 1188 & $51,8 \%$ \\
\hline Total & 3996 & $100,0 \%$ & 2292 & $100,0 \%$ \\
\hline \multicolumn{3}{|c}{ Fonte: Elaborado pela autora, 2016 }
\end{tabular}

Fonte: Elaborado pela autora, 2016

Analisando os países em que houve mais publicações, os Estados Unidos aparecem em primeiro lugar, com 531 artigos publicados, o que equivale a $28 \%$ das publicações internacionais. Em segundo lugar, o Reino Unido com $26 \%$ e a Holanda com 296 publicações, seguido por Alemanha, Venezuela, Espanha e Colômbia, que teve 2\% do total. Os 'outros' da Tabela 2 correspondem a cinquenta países, onde foram publicados 341 artigos, $18 \%$ das 1.875 publicações internacionais.

Tabela 2 - Países publicados

\begin{tabular}{lcc}
\hline \multicolumn{1}{c}{ País } & \multicolumn{2}{c}{ Artigos } \\
\hline Estados Unidos & 531 & $28 \%$ \\
Reino Unido & 484 & $26 \%$ \\
Holanda & 296 & $16 \%$ \\
Alemanha & 100 & $5 \%$ \\
Venezuela & 46 & $2 \%$ \\
Espanha & 42 & $2 \%$ \\
Colômbia & 35 & $2 \%$ \\
Outros & 341 & $18 \%$ \\
\hline Total & $\mathbf{1 8 7 5}$ & $\mathbf{1 0 0 \%}$ \\
\hline \multicolumn{2}{c}{ Fonte: Elaborado pela autora, 2016 }
\end{tabular}

Vale destacar que os países da América do Sul, como a Venezuela, a Colômbia, o Chile e a Argentina, aparecem entre os dez países com maior número de publicações. A 
Venezuela com 46 publicações, a Colômbia com 35 artigos, o Chile com 23 e a Argentina com 22. O México, país localizado na América do Norte, aparece em $13^{\circ}$ lugar com 18 publicações. Observou-se que Portugal, país de língua portuguesa, mesma oficial do Brasil, aparece na $16^{\mathrm{a}}$ posição com 17 artigos publicados, o que corresponde a menos de $1 \%$ do total de publicações científicas internacionais.

Gráfico 1 - Porcentagem de artigos por país

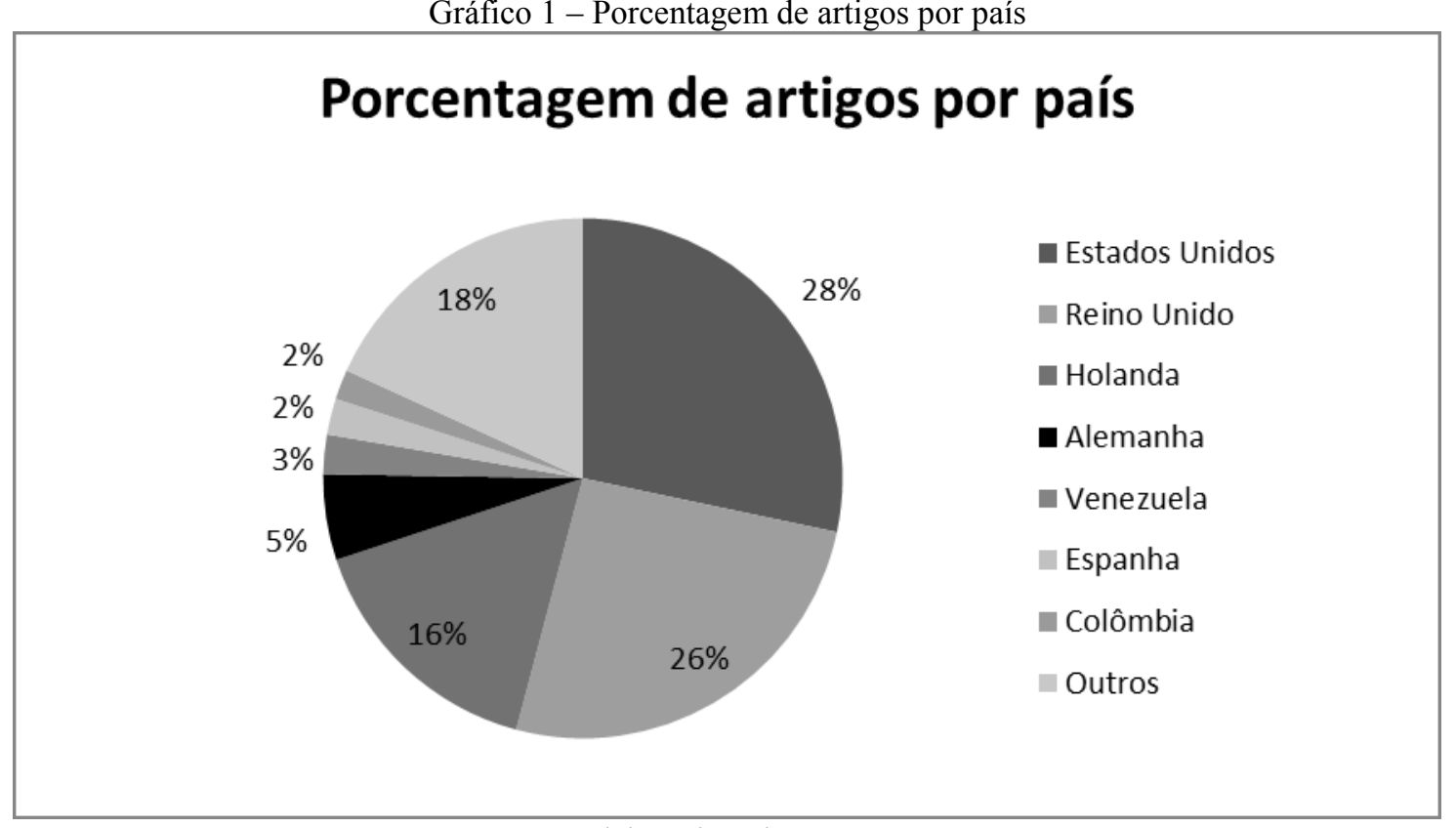

Fonte: elaborado pela autora, 2016

É importante destacar que a revista com maior número de artigos publicados foi a Espacios da Colômbia, com 32 artigos publicados em 2015. A revista Business Management Review (BMR), do Reino Unido, também aparece entre as principais fontes de publicação, com 18 artigos publicados.

Partindo para a análise das áreas temas das publicações, tomando como base a classificação da Coordenação de Aperfeiçoamento de Pessoal de Nível Superior (CAPES, 2016) para as Grandes Áreas do Conhecimento, foram considerados todos os artigos por pesquisador, para que fosse possível fazer uma análise do número de artigos publicados e da quantidade de pesquisadores por área de atuação na UFSC. Portanto, o mesmo artigo pode ser considerado para duas áreas devido à participação de mais de um autor.

Vale destacar também que $33 \%$ dos pesquisadores da UFSC atuam em mais de uma Grande Área do Conhecimento, o que equivale a 1.427 pessoas. Já, os que atuam em apenas uma das Grandes Áreas totalizam 67\% ou 2.917 dos pesquisadores da Universidade. 
Tabela 3 - Quantidade de artigo/pesquisador por área de atuação

\begin{tabular}{lcccc}
\hline Grandes Áreas & \multicolumn{1}{c}{ Artigo por Grande Área } & no de pesquisadores por área de atuação \\
\hline Ciências agrárias & 335 & $13 \%$ & 315 & $5 \%$ \\
Ciências biológicas & 458 & $18 \%$ & 439 & $8 \%$ \\
Ciências da saúde & 486 & $19 \%$ & 1082 & $19 \%$ \\
Ciências exatas e da terra & 478 & $19 \%$ & 727 & $12 \%$ \\
Ciências humanas & 97 & $4 \%$ & 1049 & $18 \%$ \\
Ciências sociais aplicadas & 157 & $6 \%$ & 986 & $17 \%$ \\
Engenharias & 512 & $20 \%$ & 744 & $13 \%$ \\
Linguística, letras e artes & 13 & $1 \%$ & 381 & $\mathbf{7 \%}$ \\
Outros & 34 & $1 \%$ & 98 & $\mathbf{2 \%}$ \\
\hline Total & $\mathbf{2 5 7 0}$ & $\mathbf{1 0 0 \%}$ & $\mathbf{5 8 2 1}$ & $\mathbf{1 0 0 \%}$ \\
\hline
\end{tabular}

Fonte: Elaborado pela autora, 2016

O maior número de publicações internacionais em 2015 foi da área de Engenharias, com 20\% do total, seguido por Ciências da Saúde (19\%) e Ciências Exatas e da Terra (19\%). Em quarto lugar ficaram as Ciências Biológicas com $18 \%$ do total de publicações internacionais.

$\mathrm{Na}$ análise comparativa do número de publicações internacionais por área com o número de pesquisadores, foi possível perceber uma discrepância entre o total de pesquisadores da área das Ciências Humanas (1.049 ou 18\%) e o número de publicações da mesma área (97 ou 4\%).

Também é possível comparar a área de Linguística, Letras e Artes que, por mais que tenha apenas $7 \%$ do número de pesquisadores (381), foi a área com menor número de publicações em 2015 (1\%), a despeito de ser a área mais propensa a escrever em outras línguas.

O gráfico abaixo evidencia as porcentagens de publicações internacionais para cada Grande Área de estudo.

Gráfico 2 - Porcentagem de artigos por área

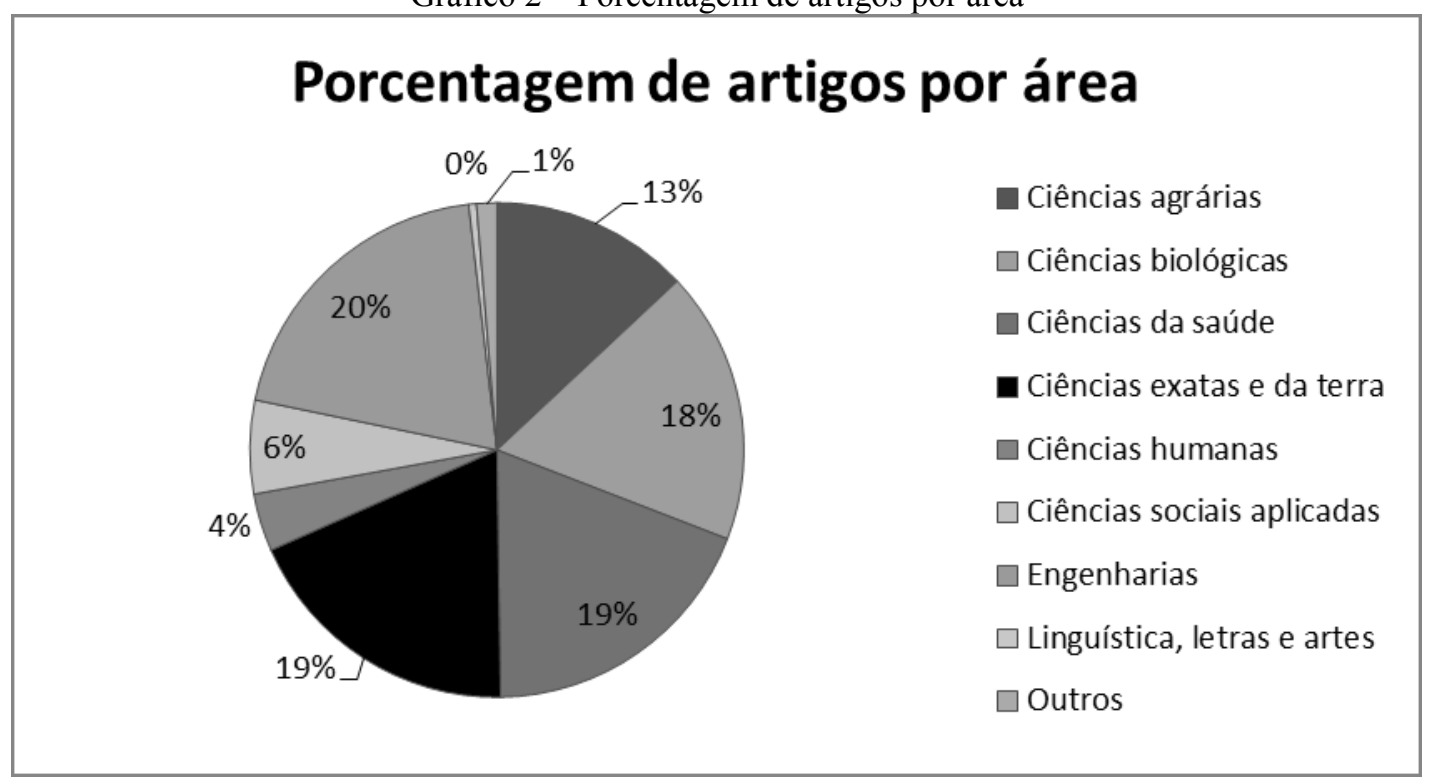

Fonte: Elaborado pela autora, 2016

A comparação entre o número de artigos publicados e o número de pesquisadores em cada Grande Área é possível ser feita a partir do gráfico a seguir, que evidencia a porcentagem, por área, do número de publicações e do número de pesquisadores. Destaca-se a 
distância entre a porcentagem de artigos das Ciências Humanas e o número de pesquisadores que atuam nessa área, 4\% para 18\%, respectivamente. $\mathrm{O}$ semelhante acontece com as Ciências Sociais Aplicadas, de 6\% para 17\%. Já, nas Ciências da Saúde, os números se equivalem, aproximadamente $19 \%$ tanto de artigos quanto de pesquisadores. Nas Ciências Biológicas ocorreu o oposto às Humanas, $18 \%$ de artigos publicados em contraponto a $8 \%$ do total de pesquisadores atuam nessa área.

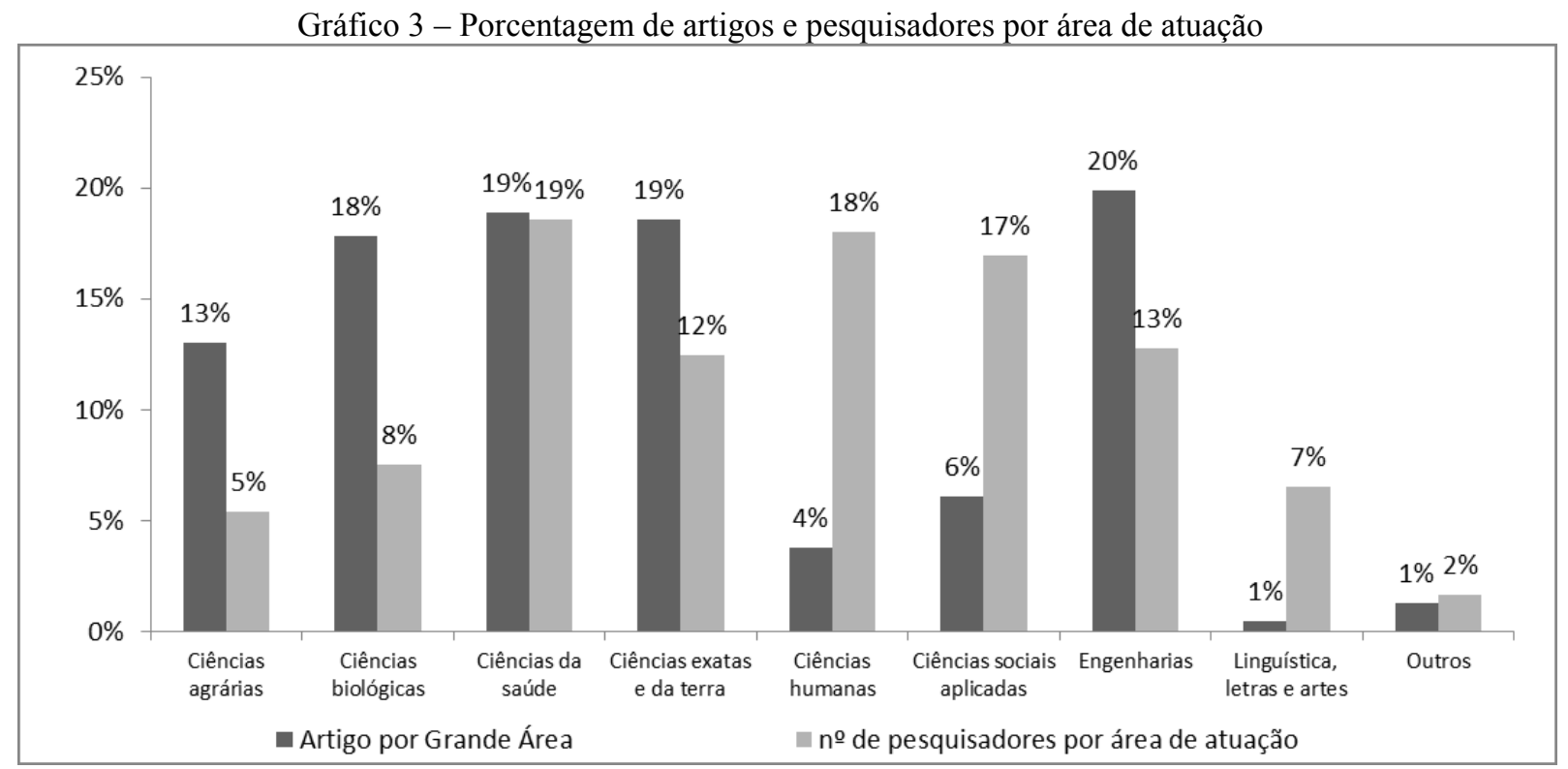

Fonte: Elaborado pela autora, 2016

Partindo-se para a análise da quantidade de artigos publicados internacionalmente por pesquisador, 446 produziram apenas um artigo em periódico internacional em 2015, seguido por 193 que publicaram dois artigos e 110 com três publicações no exterior. Como pode ser observado na Tabela 4, 29 investigadores publicaram 10 ou mais artigos, sendo que um desses destacou-se por ter publicado 25 artigos em periódicos ou revistas internacionais no ano de 2015.

\begin{tabular}{|c|c|c|}
\hline \multicolumn{3}{|c|}{ № de artigos publicados em 2015} \\
\hline 1 Artigo & 442 & $46 \%$ \\
\hline 2 Artigos & 193 & $20 \%$ \\
\hline 3 Artigos & 110 & $11 \%$ \\
\hline 4 Artigos & 61 & $6 \%$ \\
\hline 5 Artigos & 40 & $4 \%$ \\
\hline 6 Artigos & 33 & $3 \%$ \\
\hline 7 Artigos & 27 & $3 \%$ \\
\hline 8 Artigos & 12 & $1 \%$ \\
\hline 9 Artigos & 13 & $1 \%$ \\
\hline 10 ou mais Artigos & 29 & $3 \%$ \\
\hline Total & 960 & $100 \%$ \\
\hline
\end{tabular}

Fonte: Elaborado pela autora, 2016

No gráfico abaixo é possível fazer uma análise comparativa entre a quantidade de artigos publicados e a porcentagem de pesquisadores. Dos pesquisadores, $46 \%$ realizaram 
apenas uma publicação em periódico internacional, 20\% tiveram duas publicações e $11 \%$ três artigos publicados. Vale destacar que 3\% dos pesquisadores da UFSC produziram 10 ou mais artigos em periódicos internacionais.

Gráfico 4 - Quantidade de artigo por pesquisador

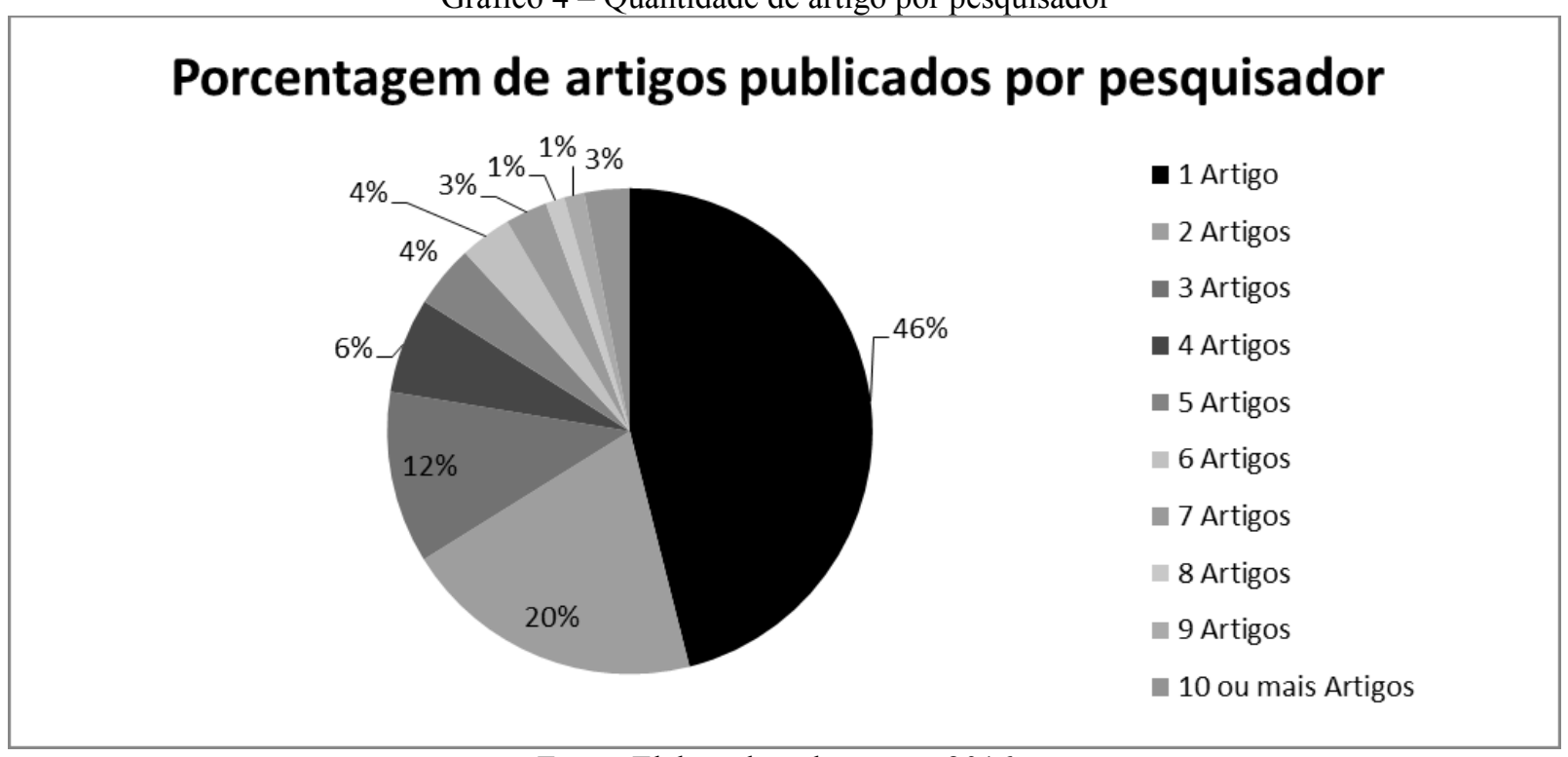

Fonte: Elaborado pela autora, 2016

É possível perceber no Gráfico 4 que um grande número de pesquisadores publicou apenas um artigo em periódico internacional durante todo o ano de 2015 , porém, vale destacar que a publicação internacional ainda ficou concentrada em poucos investigadores da Universidade Federal de Santa Catarina. Além disso, algumas áreas se destacam com um grande número de publicações, evidenciando que as demais necessitam maior atenção por parte da Universidade, já que pouco se abriram ou colaboram com o ambiente científico internacional.

\section{CONSIDERAÇÕES FINAIS}

O estudo aqui descrito teve como objetivo analisar quantitativamente a publicação científica dos pesquisadores da Universidade Federal de Santa Catarina no ano de 2015 em periódicos internacionais, após identificar a publicação internacional como fator de avaliação da internacionalização das universidades, com base nas literaturas internacional e nacional. A análise foi realizada extraindo-se as informações constantes nos currículos da Plataforma Lattes dos pesquisadores da UFSC.

Para Stallivieri (2009), com frequência, dentro das universidades, acontecem contatos internacionais feitos diretamente pelos pesquisadores, muitas vezes não envolvendo os órgãos centrais da Instituição. Por isso, em estudos como este é possível identificar um forte capital intelectual já instalado na Universidade e descobrir maiores níveis de internacionalização, para que se tornem conhecidos pela própria instituição.

Corroborando com isso, UNESCO (2003) lista alguns dos benefícios da internacionalização da educação superior, a qual é considerada tanto no mundo acadêmico como no governo uma direção para o desenvolvimento. A formação de redes acadêmicas e o desenvolvimento curricular são oportunidades advindas da internacionalização. Na pesquisa em redes, Serra, Fiates e Ferreira (2008) apontam que há interesse de pesquisadores 
americanos em desenvolver parcerias com pesquisadores latino-americanos, oportunizando maior aprendizado para todos.

Pesquisas que se destacam internacionalmente beneficiam tanto o pesquisador quanto a universidade, que pode atrair financiamento para o desenvolvimento de novas pesquisas, publicações, prêmios e citações em artigos nacionais e internacionais (Vargas, Félix, Matos, 2004). De acordo com os autores, é fundamental que a UFSC avalie as pesquisas realizadas no âmbito da universidade, para que seja possível identificar quais as áreas que mais publicam, além de analisar a qualidade das publicações. Essa avaliação torna-se fundamental no momento de distribuir recursos financeiros, estimular a capacitação, possibilitar a expansão de áreas de pesquisa e criar novos laboratórios (VARGAS, FÉLIX, MATOS, 2004).

Serra, Fiates e Ferreira (2008) defendem que há uma grande diferença entre publicar no Brasil e nas revistas internacionais, as quais são normalmente dominadas pelas publicações americanas, o que se torna um dos motivos para a pequena quantidade de artigos com autoria brasileira nos periódicos internacionais bem qualificados. Diante disso, os autores acreditam que a participação de professores e pesquisadores brasileiros em eventos internacionais é fundamental, já que possibilita a troca de experiências e a criação de parcerias com pesquisadores e instituições estrangeiras.

Ou seja, é preciso que o cotidiano da universidade envolva o apoio à pesquisa e à internacionalização. Diante disso, o presente artigo buscou chamar a atenção para a publicação científica internacional dos pesquisadores da Universidade Federal de Santa Catarina por meio da exposição das características das publicações internacionais no ano de 2015.

Foi possível compreender que o nível de internacionalização das publicações científicas de pesquisadores da UFSC é considerável, já que $46,9 \%$ dos artigos publicados foram em periódicos ou revistas internacionais, ou seja, quase a metade da produção científica elevou o nome da Universidade, projetando-a no cenário da educação e da pesquisa em nível global. Ainda assim, as publicações estão concentradas em países de língua inglesa, como os Estados Unidos e o Reino Unido. Além disso, algumas áreas têm maior número de publicação, mesmo em comparação com o número de pesquisadores por área de atuação.

Espera-se que as reflexões advindas deste artigo instiguem a busca por novos espaços para publicações, possibilidades de convênios e acordos de cooperação, pesquisas com participação internacional e patamares mais elevados para a internacionalização da Universidade Federal de Santa Catarina e de outras Instituições de Ensino Superior, as quais são influenciadas diariamente pela dinâmica da globalização.

\section{REFERÊNCIAS}

ABC. Agência Brasileira de Cooperação. Ministério das Relações Exteriores. Disponível em $<$ http://www.abc.gov.br/>. Acesso em 18 jul 2016.

BOZU, Zoia; HERRERA, Pedro José Canto. El profesorado universitario en la sociedad del conocimiento: competencias profesionales docentes. Revista de Formación e Innovación Educativa Universitaria. Vol. 2, № 2, 87-97, 2009.

CAPES. Coordenação de Aperfeiçoamento de Pessoal de Nível Superior. Disponível em $<$ http://www.capes.gov.br/>. Acesso em 18 jul 2016.

CNPQ. Conselho Nacional de Desenvolvimento Científico e Tecnológico. Disponível em $<$ http://www.cnpq.br/web/guest/competencias/>. Acesso em 18 jul 2016. 
DELGADO-MÁRQUEZ, Blanca L.; HURTADO-TORRES, Nuria Esther; BONDAR, Yaroslava. La internacionalización en la enseñanza superior: investigación teórica y empírica sobre su influencia en las clasificaciones de las instituciones universitárias. Revista de Universidad e y Sociedad del Conocimiento, vol. 8 n. ${ }^{\circ}$ 2, Universitat Oberta de Catalunya, Barcelona, julio de 2011.

GACEL, Jocelyne; AVILA, Ricardo. Universidades Latinoamericanas frente al reto de la internacionalización. Revista Casa del Tiempo, V. 1, Num 9, Pags 2-8, Guadalajara, Julho 2009.

KNIGHT, Jane. Cinco verdades a respeito da internacionalização. In: International Higher Education, Revista Ensino Superior. UNICAMP, 2012. Disponível em $<$ https://www.revistaensinosuperior.gr.unicamp.br/international-higher-education/cincoverdades-a-respeito-da-internacionalizacao>. Acesso em 30 jun 2016.

LÓPEZ, María Paz. Aportes para pensar las dimensiones internacionales de la investigación em América Latina. Revista Iberoamericana de Ciência, Tecnología y Sociedad, $\mathrm{n}^{\mathbf{0}}$ 30, v.10, Set 2015 .

MANUAL DE SANTIAGO. Manual de Indicadores de Internacionalización de la Ciencia y la Tecnología. Red Iberoamericana de Indicadores de Ciencia y Tecnología. Santiago, 2007.

MOREIRA, Herivelto; CALEFFE, Luiz Gonzaga. Metodologia da pesquisa para o professor pesquisador. 2.ed. Rio de Janeiro: Lamparina, 2008.

MOROSINI, Marilia Costa. Estado do conhecimento sobre internacionalização da educação superior - conceitos e práticas. Educar, Curitiba, n. 28, p. 107-124, 2006. Editora UFPR

PDI 2004-2009. Plano de Desenvolvimento Institucional 2004 a 2009. Universidade Federal de Santa Catarina. Florianópolis: UFSC, 2004.

PDI 2010-2014. Plano de Desenvolvimento Institucional 2010 a 2014. Universidade Federal de Santa Catarina. Florianópolis: UFSC, 2010.

PDI 2015-2019. Plano de Desenvolvimento Institucional 2015 a 2019. Universidade Federal de Santa Catarina. - Florianópolis: UFSC, 2015.

PNPG. Plano Nacional de Pós-Graduação (PNPG) 2011-2020. Vol 1. Coordenação de Pessoal de Nível Superior. Brasília: CAPES, 2010.

Relatório de Gestão 2003 UFSC. Universidade Federal de Santa Catarina. Florianópolis: UFSC, 2004.

RIBEIRO, Paulo José Gonzaga. Adaptação organizacional estratégica na instituição de ensino superior, por meio da internacionalização: o caso da Universidade Católica de Goiás. Florianópolis, UFSC, Programa de Pós-Graduação em Engenharia de Produção, 2000. 
RUDIO, Franz Victor. Introdução ao projeto de pesquisa científica. 31.ed. Petrópolis: Vozes, 2003.

SANTOS, Antonio Raimundo dos. Metodologia Científica: a construção do conhecimento. 6.ed. Rio de Janeiro: DP\&A, 2004.

SEBASTIÁN, Jesús (autor y coordinador). Estrategias de cooperación universitaria para la formación de investigadores en Iberoamérica. Madrid: Organización de Estados Iberoamericanos para la Educación, la Ciencia y la Cultura (OEI), 2003.

SERRA, Fernando A. Ribeiro; FIATES, Gabriela Gonçalves; FERREIRA, Manuel Portugal. Publicar é difícil ou faltam competências? O desafio de pesquisar e publicar em revistas científicas na visão de editores e revisores internacionais. RAM, Rev. Adm. Mackenzie, São Paulo, v. 9, n. 4, p. 32-55, June 2008. Disponível em $<$

http://www.scielo.br/scielo.php?script=sci_arttext\&pid=S1678-69712008000400004>. Acesso em 05 jul 2016.

SILVA, Darly Henriques da. Cooperação internacional em ciência e tecnologia:

oportunidades e riscos. Revista Brasileira de Política Internacional. 50 (1): 5-28, 2007.

SINTER. Secretaria de Relações Internacionais. Universidade Federal de Santa Catarina. Disponível em $<$ http://sinter.ufsc.br/sinter/>. Acesso em 19 jul 2016.

SOARES, Sandro Vieira; CASA NOVA, Silvia Pereira de Castro. Pesquisadores brasileiros que publicam em periódicos internacionais: qual sua formação acadêmica? XV Colóquio Internacional de Gestão Universitária - CIGU. Anais. Mar del Plata, 2015.

STALLIVIERI, Luciane. As Dinâmicas de uma nova Linguagem Intercultural na Mobilidade Acadêmica Internacional. Universidad Del Salvador. (Tese) Programa de Doutorado em Línguas Modernas da Universidad Del Salvador. Buenos Aires, 2009, 234f.

STALLIVIERI, Luciane. Estratégias de internacionalização das universidades brasileiras. Caxias do Sul: Educs, 2004.

UNESCO. Educação superior: reforma, mudança e internacionalização. Anais. Brasília: UNESCO Brasil, SESU, 2003.

VARGAS, Getúlio; FÉLIX, Grasiely Cachoeira; MATOS, Marilú Luiza de. Uma Análise da Evolução Quantitativa da Produção Científica da Universidade Federal de Santa Catarina. IV Colóquio Internacional sobre Gestão Universitária na América do Sul. Florianópolis, 2004.

VIEIRA, Rosilene C.; LIMA, Manolita C. Academic Ranking - From Its Genesis to Its International Expansion. Higher Education Studies, Vol.5, Nº.1, 2015. 